\title{
Matrix Encryption Scheme
}

Abdelhakim Chillali ${ }^{*}$

Sidi Mohamed Ben Abdellah University, Mathematics Physics and Computer Science, LSI, FP, Taza, Morocco

\begin{tabular}{l} 
A R T I C L E I N F O \\
\hline Article history: \\
Received: 12 April, 2017 \\
Accepted: 04 May, 2017 \\
Online: 14 May, 2017 \\
\hline Keywords: \\
Public key cryptography \\
Discrete logarithm problem \\
Finite field
\end{tabular}

A R T I C L E I N F O

Received: 12 April, 2017

Accepted: 04 May, 2017

Online: 14 May, 2017

Keywords:

Discrete logarithm problem

\section{Introduction}

Public key cryptographic is the fundamental technology in secure communications. It was devised by Diffie and Hellman in 1976 to secret key distribution. The mathematical problems more used are the discrete logarithm problem (DLP). In 1985 the elliptic curve discrete logarithm problem (ECDLP) was proposed independently by Koblitz and Miller. In this paper, we present the Matrix discrete logarithm problem in a new cryptographic scheme. Consider a finite field $L=\mathbb{F}_{q}$, where $\mathrm{q}$ is a power of $\mathrm{p}$ the characteristic of $L .[1,2,3]$

Throughout this work, we denote: $L^{*}$ multiplicative group of $L$. Let $a, b \in L^{*}$ and let $x, y \in L$,

$$
\begin{gathered}
M_{y}^{x}=\left(\begin{array}{cc}
\frac{x}{a}-1 & \frac{y}{b}-1 \\
\frac{y}{b}+1 & \frac{x}{a}+1
\end{array}\right) \\
G=\left\{M_{y}^{x} / \operatorname{det}\left(M_{y}^{x}\right)=1\right\} \\
G_{q}=G \bmod p . \\
M_{y_{1}}^{x_{1}} \Delta M_{y_{2}}^{x_{2}}=M_{y_{3}}^{x_{3}}
\end{gathered}
$$

\begin{abstract}
A B S T R A C T classical cryptography, the Hill cipher is a polygraphic substit result to a receiver. Upon receipt of the result, the receiver can retrieve the sender's clear message by performing the inverse calculation.
\end{abstract}

where,

$$
\begin{aligned}
& \text { (1): }\left\{\begin{array}{l}
x_{3}=\frac{b^{2} x_{1} x_{2}+a^{2} y_{1} y_{2}}{a b^{2}} \\
y_{3}=\frac{x_{1} y_{2}+x_{2} y_{1}}{a}
\end{array}\right. \\
& M_{k}=\left(\begin{array}{cc}
\frac{k^{2}+1}{2 k}-1 & \frac{k^{2}-1}{2 k}-1 \\
\frac{k^{2}-1}{2 k}+1 & \frac{k^{2}+1}{2 k}+1
\end{array}\right), k \in L^{*} . \\
& N_{1}^{\Delta k_{1}} \Delta N_{2}^{\Delta k_{2}} \Delta \ldots \ldots . . \Delta N_{\theta(\log q)}^{\Delta k_{\theta(\log q)}}=M
\end{aligned}
$$

$$
m=\left|G_{q}\right|
$$

The next theorem whose proof is evident.

Theorem 1 The set $G_{q}$ with the operator $\triangle$ defined by (1) is a abelian group.

The identity element is $M_{0}^{a}$, that if $M=M_{y}^{x}$ then $N=$ $M_{-y}^{x}$ is the invertible element of $M$.

Remark 1 The MDLP consists of following for two elements $M, N \in G_{q}$, determine the scaler $k \in \mathbb{Z}_{m}$ such that $M^{\Delta k}=N$. It is necessary that $M$ be a generator of the group $G_{q}$.

Assumption 1 Given a group $G_{q}$ and tow elements $M$ and $N \in G_{q}$, there exists non polynomial time algorithm $\theta(\log q)$ deciding the integer $k$ such that $M^{\Delta k}=N$ if such a $k$ exists.

\footnotetext{
*Abdelhakim Chillali , FP , Taza, Morocco \& abdelhakim.chillali@usmba.ac.ma
} 


\section{Matrix Cryptosystem}

\subsection{Key distribution protocols}

Let $M_{y}^{x}$ be a generator of the group $G_{q}$.

Alice take a private key $1<l<m$, and computes $M_{y_{l}}^{x_{l}}=$ $M_{y}^{x \Delta l}$, then she transmits $M_{y_{l}}^{x_{l}}$ to Bob.

Similar, Bob takes a private kev $1<t<m$, and computes $M_{y_{t}}^{x_{t}}=M_{y}^{x \Delta t}$ and transmits $M_{y_{t}}^{x_{t}}$ to Alice.

In the same way Alice and Bob compute $M_{y_{t l}}^{x_{t l}}=M_{y_{t}}^{x_{t} \Delta l}$ and $M_{y_{l t}}^{x_{l t}}=M_{y_{l}}^{x_{l} \Delta t}$ respectively.

\section{Theorem 2}

$$
\frac{x_{l t}}{a}+\frac{y_{l t}}{b}=\frac{x_{t l}}{a}+\frac{y_{t l}}{b} \bmod p
$$

The secret key is $\alpha=\frac{x_{t l}}{a}+\frac{y_{t l}}{b} \bmod p$

\subsection{Description of This Cryptosystem}

Let $L=\mathbb{F}_{q}$ with $q=p^{n}$.

1)Space of lights: $P=G_{q}$.

2)Space of quantified: $C=G_{q}$.

3) Space of the keys: $K=L^{*}$.

4)Function of encryption: $\forall \alpha \in K$,

$$
e_{\alpha}: \mid \begin{array}{rll}
P & \longrightarrow C \\
M_{y}^{x} & \longmapsto e_{\alpha}\left(M_{y}^{x}\right)=M_{y}^{x} \Delta M_{\alpha}
\end{array}
$$

5)Function of decryption: $\forall \alpha \in K$,

$$
d_{\alpha}: \mid \begin{array}{rll}
C & \longrightarrow P \\
M_{y}^{x} & \longmapsto d_{\alpha}\left(M_{y}^{x}\right)=M_{y}^{x} \Delta M_{-\alpha}
\end{array}
$$

\section{Remark 2}

$$
d_{\alpha} o e_{\alpha}\left(M_{y}^{x}\right)=M_{y}^{x} \Delta M_{\alpha} \Delta M_{-\alpha}=M_{y}^{x}
$$

Remark 3 a) Secret key : $\alpha$

b) Public keys:

1) Space of lights; $P$

2) Space of quantified; $C$

3) Space of the keys; $K$

4) Generator of the group $P ; M_{y}^{x}$

5) Function of encryption; $e_{\alpha}$

6) Function of decryption; $d_{\alpha}$

Remark 4 The $M_{y_{l}}^{x_{l}}, M_{y_{t}}^{x_{t}}$ and $m$ are public and can known by another person, but to obtain the private key $\alpha$, it is necessary to solve the Matrix problem discrete logarithm in $G_{q}$, what returns the discovery of the difficult key $\alpha$.

\subsection{Numerical Example}

Alice and Bob Choose the following public numbers; $p=41, a=2, b=5$, and $n=1$. They determine the group $\left.G_{41}=<M_{31}^{26}\right\rangle$, with the identity element $M_{0}^{2}$.
1) Exchange of the key deprived between Alice and Bob:

Alice take a private key; $l=13<39$, calculation; $M_{23}^{13}=$ $M_{31}^{26 \Delta 13}$ and send to Bob $M_{23}^{13}$. In turn, Bob take a private key; $t=21<39$, calculation; $M_{10}^{15}=M_{31}^{26 \Delta 21}$ and send it to Alice. Alice and Bob calculate separately: $M_{18}^{13}=M_{10}^{15 \Delta 13}$ and $M_{18}^{13}=M_{23}^{13} \Delta 21$. They determine their secret key:

$$
\alpha=\frac{13}{2}+\frac{18}{5}=6 \bmod 41
$$

2) Message to send:

Alice wants to send the following message

$$
m e=\left\{M_{18}^{28}, M_{4}^{0}, M_{27}^{23}, M_{36}^{34}\right\}
$$

It encrypts it using the encryption function

\begin{tabular}{|c|c|}
\hline$M_{y}^{x}$ & \multicolumn{2}{|c|}{$e_{6}\left(M_{y}^{x}\right)$} \\
\hline$M_{18}^{28}$ & $\left(\begin{array}{cc}39 & 40 \\
1 & 0\end{array}\right)$ \\
\hline$M_{4}^{0}$ & $\left(\begin{array}{cc}15 & 37 \\
39 & 17\end{array}\right)$ \\
\hline$M_{27}^{23}$ & $\left(\begin{array}{cc}28 & 13 \\
17 & 30\end{array}\right)$ \\
\hline$M_{36}^{34}$ & $\left(\begin{array}{ll}28 & 25 \\
27 & 30\end{array}\right)$ \\
\hline
\end{tabular}

3) Message received:

Bob receives message crypt send by Alice

$m r=\left\{\left(\begin{array}{ll}28 & 25 \\ 27 & 30\end{array}\right),\left(\begin{array}{ll}28 & 13 \\ 17 & 30\end{array}\right),\left(\begin{array}{cc}15 & 37 \\ 39 & 17\end{array}\right),\left(\begin{array}{cc}39 & 40 \\ 1 & 0\end{array}\right)\right\}$

It decrypts it using the decryption function

\begin{tabular}{|c|c|}
\hline$M_{y}^{x}$ & $d_{6}\left(M_{y}^{x}\right)$ \\
\hline$\left(\begin{array}{cc}39 & 40 \\
1 & 0\end{array}\right)$ & $M_{18}^{28}$ \\
\hline$\left(\begin{array}{cc}15 & 37 \\
39 & 17\end{array}\right)$ & $M_{4}^{0}$ \\
\hline$\left(\begin{array}{cc}28 & 25 \\
27 & 30\end{array}\right)$ & $M_{36}^{34}$ \\
\hline$\left(\begin{array}{cc}28 & 13 \\
17 & 30\end{array}\right)$ & $M_{27}^{23}$ \\
\hline
\end{tabular}

\section{Example for cryptography}

In this example we take $p=3, a=b=1, n=3$, and $\alpha$ root of the polynomial $X^{3}+2 X+1$. We have $P=G_{27}$, $C=G_{27}$, and $K=\mathbb{F}_{27}^{*}$ and $M_{\alpha^{2}}^{2 \alpha+2}$ is a generator of the group $P$.

1) Exchange of the key deprived: Alice take a private key $l=12<25$, send to Bob $M_{\alpha^{2}}^{\alpha+1}=$ $M_{\alpha^{2}}^{2 \alpha+2 \Delta l}$. Bob take a private key $t=20<25$, send to Alice $M_{2 \alpha^{2}+\alpha+1}^{2 \alpha^{2}+2}=M_{\alpha^{2}}^{2 \alpha+2 \Delta t}$. Their secret key is

$$
\beta=2 \alpha^{2}+2+\alpha^{2}+2 \alpha+2=2 \alpha+1 .
$$


2) Message Encryption:

It is known that the encryption functions and the decryption functions are defined by:

$$
\begin{aligned}
& e_{\beta}\left(M_{y}^{x}\right)=M_{y}^{x} \Delta M_{\alpha^{2}+2 \alpha+2}^{2 \alpha^{2}+2} \\
& d_{\beta}\left(M_{y}^{x}\right)=M_{y}^{x} \Delta M_{2 \alpha^{2}+\alpha+1}^{2 \alpha^{2}+2}
\end{aligned}
$$

Lets $x=i \alpha^{2}+j \alpha+k$ and $y=l \alpha^{2}+m \alpha+n$, we denote $M_{y}^{x}$ by ijklmn.

Each letter is represented by a ijklmn character. Often the simple The scheme $a=001000, b=010112, \ldots$, $z=221102$ is used, but this is Not an essential feature of encryption. To encrypt a message, each letter will be decrypted by the decryption function, for a message one obtains a block of $n$ letters (considered as an n-component vector). Consider the message 'bonjour' Which will be encrypted by the message: "crvzrng".

Table of the Symbol Encryption

\begin{tabular}{|c|c|c|c|}
\hline$M_{y}^{x}$ & Symbol & $e_{\beta}\left(M_{y}^{x}\right)$ & Encrypt Symbol \\
\hline 001000 & $a$ & 202120 & $h$ \\
\hline 010112 & $b$ & 010220 & $c$ \\
\hline 010220 & $c$ & 012210 & $d$ \\
\hline 012210 & $d$ & 211110 & $s$ \\
\hline 012122 & $e$ & 010112 & $b$ \\
\hline 122110 & $f$ & 021210 & $q$ \\
\hline 122222 & $g$ & 112102 & $m$ \\
\hline 202120 & $h$ & 011101 & $j$ \\
\hline 202212 & $i$ & 001000 & $a$ \\
\hline 011101 & $j$ & 221102 & $z$ \\
\hline 011201 & $k$ & 202212 & $i$ \\
\hline 112200 & $l$ & 122110 & $f$ \\
\hline 112102 & $m$ & 022101 & $w$ \\
\hline 002001 & $n$ & 101212 & $v$ \\
\hline 020112 & $o$ & 021122 & $r$ \\
\hline 020220 & $p$ & 020112 & $o$ \\
\hline 021210 & $q$ & 020220 & $p$ \\
\hline 021122 & $r$ & 122222 & $g$ \\
\hline 211110 & $s$ & 221200 & $y$ \\
\hline 211222 & $t$ & 012122 & $e$ \\
\hline 101120 & $u$ & 002001 & $n$ \\
\hline 101212 & $v$ & 022201 & $x$ \\
\hline 022101 & $w$ & 101120 & $u$ \\
\hline 022201 & $x$ & 112200 & $l$ \\
\hline 221200 & $y$ & 011201 & $k$ \\
\hline 221102 & $z$ & 211222 & $t$ \\
\hline & & & \\
\hline
\end{tabular}

\section{Conclusion}

Although matrix multiplication can not provide security for the encryption of a message $[4,5,6]$, we have been able to construct a law of internal composition other than the law of multiplication, which allows us to create a cryptography on the matrices and which is safer for a key of reasonable length.

\section{References}

1. A. Chillali, "Cryptography over elliptic curve of the ring $F q[e], e^{4}=0$.", World Academy of Science, Engineering and Technology., 78, 848-850, 2011.

2. A. Tadmori, A. Chillali, M. Ziane, "The binary operations calculus in $E_{a, b, c}$. ", International Journal of Mathematical Models and Methods in Applied Sciences., 9, 171-175, 2015.

3. A. Tadmori, A. Chillali, M. Ziane, "Elliptic Curve over Ring $A_{4}$.", Applied Mathematical Sciences., 35(9), 1721-1733, 2015.

4. Lester S. Hill, "Cryptography in an Algebraic Alphabet.", The American Mathematical Monthly., 36, 1929.

5. Lester S. Hill, "Concerning Certain Linear Transformation Apparatus of Cryptography.", The American Mathematical Monthly., 38(9), 1931.

6. Christos Koukouvinos and Dimitris E. Simos, "Encryption Schemes based on Hadamard Matrices with Circulant Cores.", Journal of Applied Mathematics and Bioinformatics., 1(3), 17-41, 2013. 